\title{
Numerical Analysis of Dielectric Heating in Cancerous Liver Tissue
}

\author{
Abdelghaffar A. Abdelmalik, ${ }^{1 *}$ Godswill Samuel Nnamdi ${ }^{1}$ and Ismail H. Zubairu ${ }^{2}$ \\ ${ }^{1}$ Department of Physics, Ahmadu Bello University, 810001 Zaria, Nigeria \\ ${ }^{2}$ Department of Radiotherapy and Oncology, Ahmadu Bello University Teaching \\ Hospital, 810001 Zaria, Nigeria \\ *Corresponding author: aaabdelmalik@gmail.com
}

Published online: 15 November 2017

To cite this article: Abdelmalik, A. A., Nnamdi, G. S. \& Zubairu, I. H. (2017). Numerical analysis of dielectric heating in cancerous liver tissue. J. Phys. Sci., 28(3), 1-17, https://doi.org/10.21315/jps2017.28.3.1

To link to this article: https://doi.org/10.21315/jps2017.28.3.1

\begin{abstract}
Percutaneous energy-based ablation has been used for the treatment of many tumour types. The advent of thermoablative technology for the treatment of small size liver tumour introduced several advantages over surgical resection. The technology involves the creation of an electromagnetic field through an intratumourally placed antenna. A process known as dielectric heating elevates the temperature of the tissue immediately surrounding the slot of the antenna where most of the coagulative necrosis occurs. Attenuation of the electromagnetic field as it travels through the tissue leads to decrease in the rate of tissue necrosis creating a zone of sub-lethal hyperthermia, and then a zone of tissue unaffected by ablation. The temperature spreads across the tissue to destroy the cancer cells. This occurs both directly and indirectly at the membrane and sub cellular levels. Several mechanisms including mitochondrial dysfunction, changes in cell membrane integrity and inhibition of DNA replication have been postulated as pathways through which cell death following hyperthermia occurs. The absorption of power by the tissue (SAR) and the destruction of cancer cell is highly dependent on time and the input power of the antenna. This paper presents the numerical analysis of the important parameters in microwave thermotherapy and the biological mechanisms involved in the death of tumour cells.
\end{abstract}

Keywords: Microwave thermotherapy, antenna, thermal field, dielectric heating, tissue necrosis

\section{INTRODUCTION}

Carcinoma is an early stage cancer that is still confined to the organ or layer, or tissue of origin. Hepatocellular carcinoma is a type of cancer that begins in the liver. It is sometimes called hepatic cancer or liver tumour. Liver tumour 
ablation involves destroying liver tumour with the local application of extreme temperatures. This induces irreversible cell injury and ultimately a programmed sequence of events leading to the elimination of dead tumour cells and coagulative necrosis. Liver thermoablation techniques include radiofrequency ablation (RFA) and microwave ablation (MWA). Percutaneous energy-based ablation has been used for the treatment of many tumour types, including liver, kidney, lung and bone cancers, as well as soft-tissue tumours of the breast, adrenal glands, and head and neck.

The advent of thermoablative technology introduced several advantages over surgical resection. These include lower morbidity, increased preservation of surrounding tissues, reduced cost and shorter hospitalisation times, as well as intraprocedural monitoring by visualisation, etc. This complementary treatment is called hyperthermia. The basic idea behind this treatment is to create an electromagnetic field (typically between 900 and $2500 \mathrm{MHz}$ ) through an intratumourally placed antenna. ${ }^{1}$ This field makes the polar molecules with permanent dipoles in the tissue undergo realignment with the oscillating electric field. These dipoles are unable to follow the rapid reversals in the field. The alignment of the molecules leads to increase in kinetic energy thereby dissipating heat into the surrounding tissue. This process, referred to as dielectric heating, elevates the temperature of the tissue. ${ }^{2,3}$

The important properties of the tissue for MWA are relative permittivity and effective conductivity. Relative permittivity describes how well a tissue will accept an electric field while the effective conductivity describes the ability of a tissue to absorb microwave energy. High-conductivity tissues have high water content and readily absorb microwaves, while low-conductivity tissues have low water content and readily allow microwave propagation. ${ }^{4}$ MWA is more suitable for tissues with high impedance, including lung and bone, and for tissues with high water content, such as solid organs and tumours. ${ }^{1,5}$

Normal tissues and cancer tissues have different conductivity. The ex vivo electrical conductivity of normal human liver and metastatic tumour tissue using the fourelectrode method shows that the conductivity of tumour tissue is significantly higher than that of normal. ${ }^{6}$ Microwave power is absorbed much more strongly by water due to its large dielectric loss at $\mathrm{GHz}$ frequencies. The loss tangent of fluids, which may be related to the ability of the fluid to absorb energy in a microwave cavity depends on the relaxation times of the molecules. MWA has the advantage of the ability to heat tissue up to $2 \mathrm{~cm}$ away from the antenna at certain frequencies. ${ }^{1}$

Research has shown that RFA, MWA and high intensity focused ultrasound (HIFU) cause focal hyperthermic injury to ablated cells. This alters the tumour 
microenvironment and causes tumour destruction to occur in two phases, through direct and indirect mechanisms. ${ }^{7}$ The direct mechanism involves the heat injury that occurred during thermal ablation process and is predominately determined by the total energy delivered to the targeted tumour. The indirect mechanism involves injury that usually occurs after thermal ablation. This produces a progression in tissue damage. The indirect injury is not well defined compared with the primary direct effects.

Several papers have reported numerical simulation of microwave ablation therapy for cancer treatment using mathematical algorithms and software packages. ${ }^{8-12}$ But the recently updated Bioheat transfer module in COMSOL 5.1 package makes possible the simulation of necrosed tissues as a function of the variable parameters. This model gives room for the approximation of several functions. These include input power versus level of necrosis and frequency versus region of maximum necrosis. These variables can be approximated using polynomials, exponential or logarithmic functions.

This paper presents a simulated thermotherapy model and analysis of heat source distribution and temperature distribution in liver tissue and how it influences tissue necrosis based on direct heat injury. The specific absorption rate (SAR) of the tissue was also analysed. The impact of varying input power and frequency on tissue necrosis was always evaluated. It also presents the biological mechanisms involved in the death of tumour cells.

\section{EXPERIMENTAL}

\subsection{Heating Method}

A thin probe as shown in Figure 1 is inserted into the region under study to generate heat on the region under study. The probe is composed of a thin flexible coaxial cable. It has an outer conductor with diameter $1.6 \mathrm{~mm}$. The inner conductor protrudes out of the probe with a length of $3 \mathrm{~mm}$. The antenna is enclosed in a sleeve (catheter) made of polytetrafluoroethylene (PTFE). The probe is inserted into the tumour and operated at $2.45 \mathrm{GHz}$. The microwave energy generated by the antenna is absorbed by the tumour. The polar molecules with permanent dipoles in the tissue will attempt to realign in the direction of the oscillating electric field. These dipoles are unable to follow the rapid reversals in the field as a result of power dissipating in the tissue due to phase lag. The rapid vibration of the molecules could lead to friction between the atoms and heat is dissipated. This is used to kill cancer cells. 


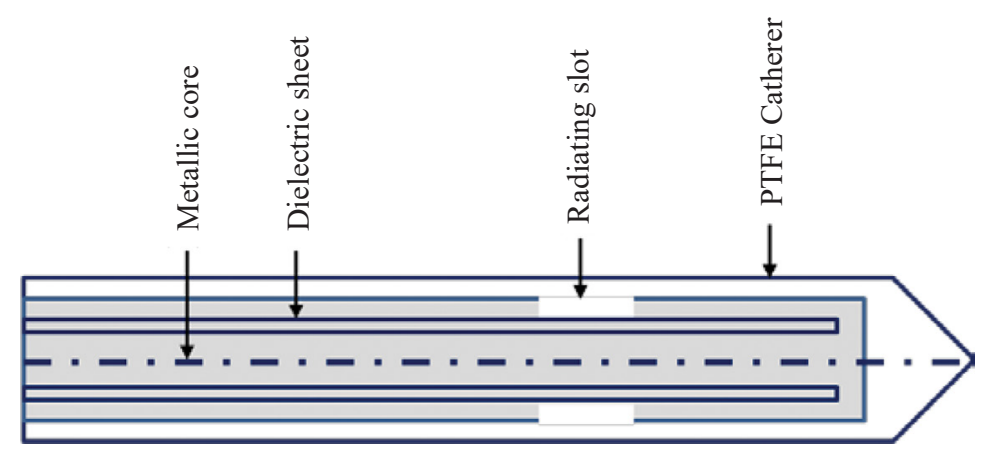

Figure 1: Schematic diagram of microwave antenna (not to scale).

The heat distribution inside the liver tissue during the thermotherapy is obtained by solving the Pennes' bioheat equation. ${ }^{13}$ The simplified form of the equation is expressed as:

$$
\rho_{t i} C_{t i} \frac{\partial T_{t i}}{\partial t}=\nabla \cdot k_{t i} \nabla T_{t i}+P_{p}+P_{e}+P_{m}
$$

where $\rho_{t i}, C_{t i}, T_{t i}$ and $k_{t i}$ are, respectively, the density, specific heat, temperature and thermal conductivity of the tissue. $P_{e}$ is the absorbed energy due to resistive heat generated by the electromagnetic field in the tissue, $P_{m}$ is the metabolic heat generation and $P_{p}$ is the perfusion heat term expressed as:

$$
P_{p}=\rho_{b l} C_{b l} \omega_{b l}\left(T_{a r t}-T_{t i}\right)
$$

where $T_{a r t}$ is the temperature of the arterial blood, and $\rho_{b l}, C_{b l}$, and $\omega_{b l}$ are, respectively, the density, specific heat, and perfusion rate of blood. The absorption of EM field gives rise to polarisation of the tissue. If the total loss in the tissue, $\sigma$ is visualised as a combination of DC conductivity, $\sigma_{0}$ of the tissue and loss due to polarisation in the tissue, $\omega \varepsilon^{\prime \prime}$ i.e.:

$$
\sigma=\omega \varepsilon^{\prime \prime}+\sigma_{0}
$$

where $\varepsilon^{\prime \prime}$ in this context is the loss due to polarisation and $\sigma_{0}=i \omega \varepsilon^{\prime}$ then the related conductivity due to polarisation is expressed as:

$$
\omega \varepsilon^{\prime \prime}=\sigma-i \omega \varepsilon^{\prime}=\sigma^{\prime}
$$

The absorbed energy due to resistive heat generated by the electromagnetic field in the tissue, $\mathrm{q}_{\mathrm{e}}$ is expressed as:

$$
P_{e}=\frac{1}{2} \sigma^{\prime}|\vec{E}|^{2}
$$


Normalisation of $P_{e}$, the absorbed energy due to resistive heat generated by the electromagnetic field in the tissue with the tissue density is referred to as the specific absorption rate (SAR):

$$
S A R=\frac{\sigma^{\prime}}{2 \rho}|\vec{E}|^{2}
$$

SAR represents the electromagnetic power deposited per unit mass in tissue $(\mathrm{W} / \mathrm{kg})$. Careful examinations of both the SAR pattern and frequency dependent reflection coefficient in tissue are essential for the optimisation of antennas for hepatic MWA.

The influence of temperature on the rate of physico-chemical reactions is often interpreted in terms of what is now known as the Arrhenius equation. The rate of tissue necrosis can be evaluated from studying the influence of temperature on the rate of tissue damage during thermotherapy. The relationship between tissue injury, $\beta$ and temperature during the ablation process can be fitted into Arrhenius relation as: ${ }^{14}$

$$
\frac{d B}{d t}=A \exp -\left(\frac{E}{\mathrm{RT}}\right)
$$

where the pre-exponential factor, $A$ is the frequency factor $\left(\mathrm{s}^{-1}\right)$ and $E$ is the activation energy for irreversible damage reaction $(\mathrm{J} / \mathrm{mol})$. These two parameters are independent of the tissue type. The fraction of necrotic tissue, $F_{n}$, is then evaluated from the relation: ${ }^{.15}$

$$
\mathrm{F}_{\mathrm{n}}=1-\exp (-\beta)
$$

\subsection{Modelling}

The model was implemented using COMSOL Multiphysics 5.1. The computer program treats simulations that involve multiple physical models or multiple simultaneous physical phenomena. It utilises finite element method (FEM) to predict induced field of realistic shape and composition. The FEM transforms differential equations over a volume to algebraic equations at the points, called nodes. These represent the solution to the governing equations and the boundary conditions in an average sense by piecewise simple functions. A modelling domain with radial and axial dimensions of $0.03 \mathrm{~m}$ and $0.08 \mathrm{~m}$ was used for the simulation. This specified the material under study. The material referred to liver in this study. An antenna was modelled with the diameter of the central conductor, inner diameter of the outer conductor and outer diameter of the outer conductor was modelled to be $0.29 \mathrm{~mm}$, $0.94 \mathrm{~mm}$ and $1.19 \mathrm{~mm}$ respectively. The diameter of the catheter was $1.79 \mathrm{~mm}$. 
The inner and the outer conductors of the antenna were modelled using perfect electric conductor boundary conditions (i.e., electric field, $\mathrm{E}=0$ ). Low-reflecting boundary conditions were used along the model boundaries to prevent reflection artefacts and axial symmetry boundary conditions were also employed along the axis of rotation. ${ }^{12}$ The relative permittivity of the inner dielectric of coaxial cable, catheter and liver tissue are 2.03, 2.60 and 43.03 respectively. The electrical conductivity of liver tissue is $1.69 \mathrm{~S} / \mathrm{m}$. ${ }^{9}$ The appropriate physics of electromagnetics and heat transfer was added to the model. The boundary condition and domains for each phenomenon was specified.

An electromagnetic wave propagating in a coaxial cable is characterised by transverse electromagnetic fields (TEM). The time-averaged power flow in the cable of impedance is:

$$
\vec{P}_{a v}=\hat{z} \pi \frac{c^{2}}{z} \ln \left(\frac{r_{\text {outer }}}{r_{\text {inner }}}\right)
$$

Using transverse magnetic (TM) formulation, the boundary condition for the metallic surfaces is:

$$
\hat{n} \times \vec{E}=0
$$

The physical and dielectric properties of the materials used for the modelling was selected from several literatures. ${ }^{11-13}$ The blood perfusion rate, $\omega_{b}=0.0036 \mathrm{~s}^{-1}$. The specific heat capacity of blood, $C_{b}$ is $3639 \mathrm{~J} / \mathrm{kg} \cdot \mathrm{K}$ and it enters the liver at the body temperature $T_{b}=37^{\circ} \mathrm{C}$ and is heated to a temperature, T. The blood has a density of $1000 \mathrm{~kg} / \mathrm{m}^{3}$ while the thermal conductivity of liver is $0.56 \mathrm{~W} / \mathrm{m} . \mathrm{K}$. The microwave frequency and input power were varied from $900 \mathrm{~Hz}$ to $2.6 \mathrm{GHz}$ and $2 \mathrm{~W}$ to $15 \mathrm{~W}$ respectively.

This model considers the heat transfer problem only in the domain of the liver. Where this domain is truncated, it uses insulation:

$$
\hat{n} \cdot \nabla T=0
$$

The volume under consideration is solved by first dividing into elements. If sufficient continuity conditions are met, the solution converges to the exact value as the elements are made smaller and increased over the volume. Free triangular mesh of maximum element size of $3 \mathrm{~mm}$ and the minimum element size of $2.5 \times 10^{-8} \mathrm{~mm}$ was used. The choice of finer mesh as shown in Figure 2 is to reduce the error of computation, thus increasing the accuracy of the result obtained. The study was done at a frequency of $50 \mathrm{~Hz}$ and for $5 \mathrm{~min}$ to $10 \mathrm{~min}$ in $10 \mathrm{~s}$ intervals. 

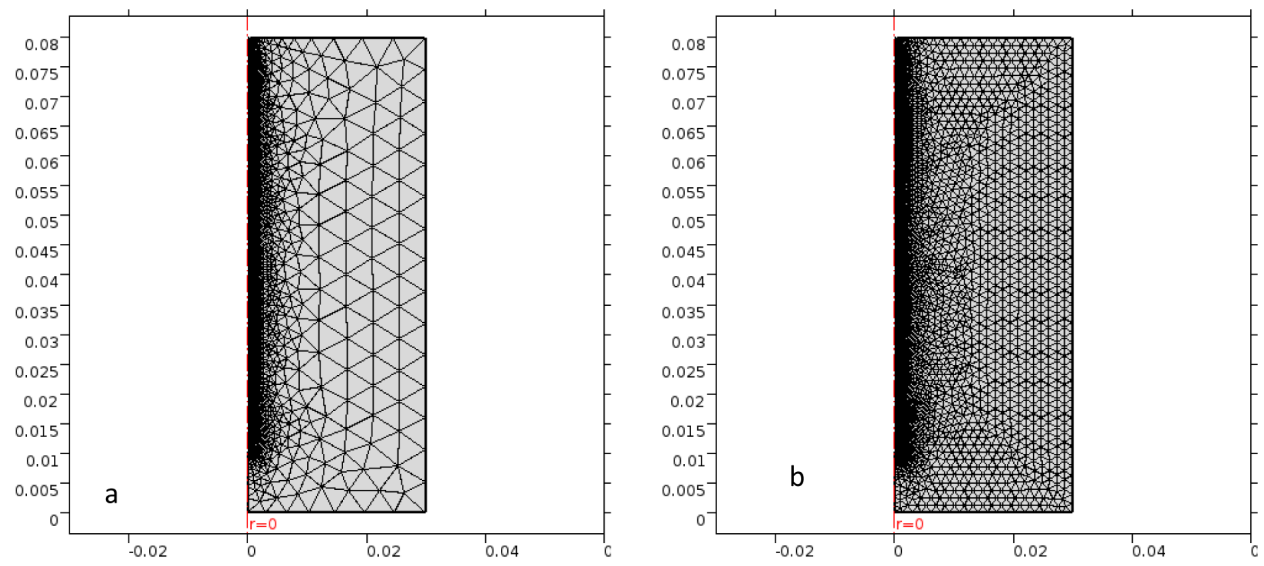

Figure 2: Illustrations of (a) default grid mesh and (b) extra fine grid mesh.

\section{RESULTS AND DISCUSSIONS}

Microwave radiation, unlike $\mathrm{X}$-ray, is a non-ionising radiation with enough energy to cause vibration of atoms in a molecule, but not enough to ionise. The electromagnetic wave distribution results in the dielectric heating. Temperature distribution has high intensity within the vicinity of the coaxial cable. The resulting steady-state temperature distribution in the liver tissue for an input microwave power of $10 \mathrm{~W}$ for the duration of $10 \mathrm{~min}$ is shown in Figure 3. The temperature distribution has a near ellipsoidal shape around the slot with the highest value near the antenna slot. It then decreased with distance from the antenna in accordance with inverse-square law. It decreased to a temperature of about $37^{\circ} \mathrm{C}$ towards the outer boundaries of the computational domain.

The microwave energy absorbed by the liver tissue attenuates due to energy absorption. The quantum energy of the microwave photons is converted to thermal energy. The thermal energy increases with time thereby leading to increase in the liver temperature. The study of the distribution of temperature in the liver shows that temperature increases with increasing time. Maximum temperature of $98.9^{\circ} \mathrm{C}$ was obtained after $5 \mathrm{~min}$ and after $10 \mathrm{~min}$, the maximum temperature increased to $103^{\circ} \mathrm{C}$. The isothermal contours at time $\mathrm{t}=10 \mathrm{~min}$ is shown in Figure 4.

Comparison of Figure 4 with Figure 5 shows that the temperature field follows the heat-source distribution. This result shows high temperatures around the antenna slot where the heat source is highest while far from the antenna, the heat source is weaker due to attenuation thereby making the blood to be able to keep the tissue at normal body temperature. 


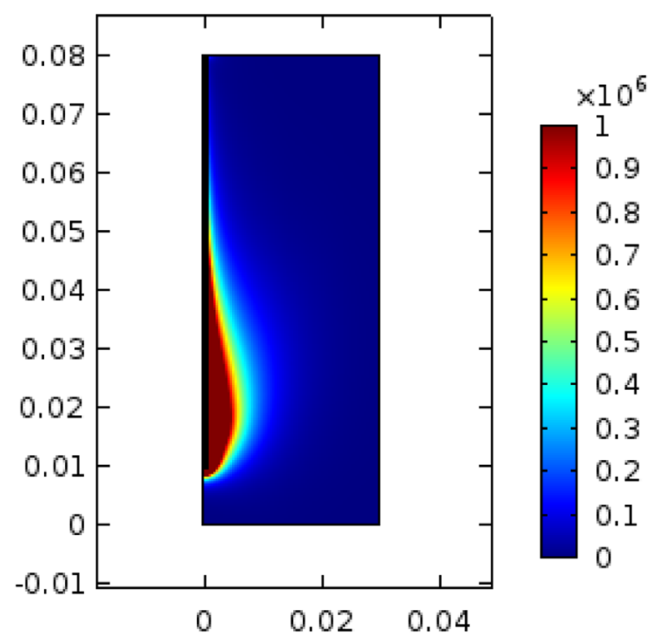

Figure 3: Computed microwave heat source density after $10 \mathrm{~min}$.

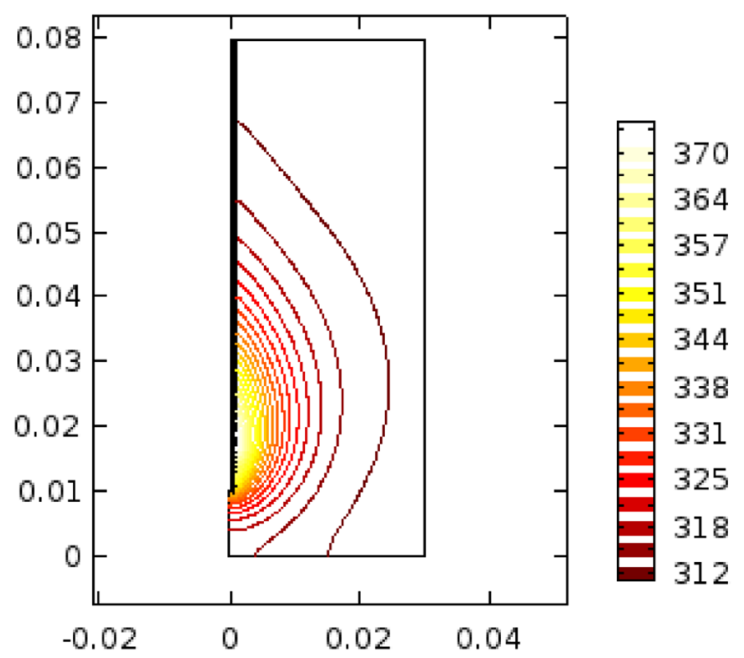

Figure 4: Isothermal contours of the liver tissue at $10 \mathrm{~min}$.

The computed power dissipated is $9.353 \mathrm{~W}$. This implies that just about $6.47 \%$ of the input $10 \mathrm{~W}$ power did not take part in the heating. Upon penetrating the tissue, the wave is attenuated and the power dissipated after passing through the distance $x$ is shown in Figure 5. It nearly satisfied the exponential relation $P=P_{0} e^{-2 a x}$ with attenuation coefficient, $\alpha=147.65$. The wave attenuation and corresponding power dissipation leads to a decrease in tissue temperature. It also has an exponential relationship as shown in Figure 6. Analysis of temperature change with time at four 
different points in the liver tissue revealed an exponential increase in temperature with time at every point. The elevated temperature resulted from the continuous increase in kinetic energy due to rotation of the molecules. As the time increases, more power is dissipated resulting in increase in temperature with time.

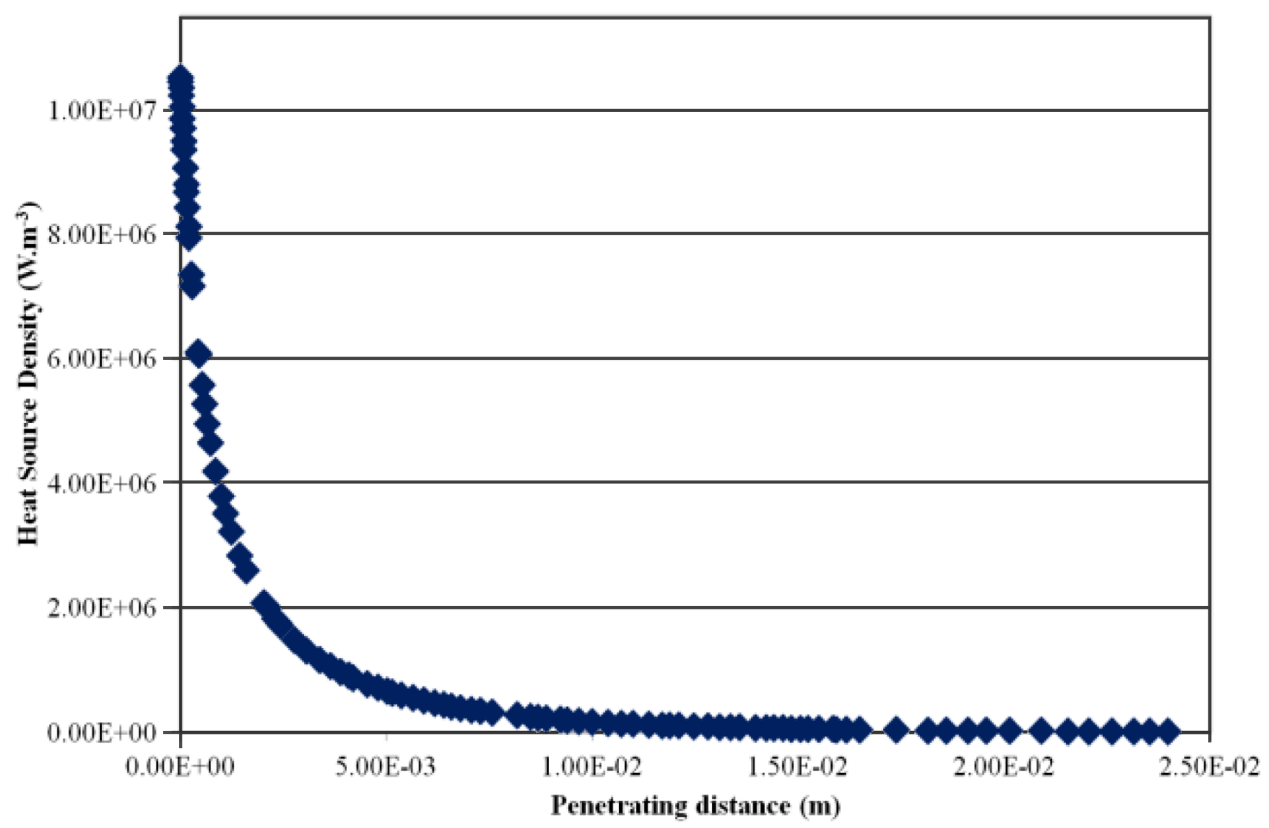

Figure 5: Power density versus penetrating distance.

Direct injury depends on the thermal energy that is applied, the rate of application and the thermal sensitivity of the target tissue. ${ }^{7}$ It occurs either by inactivation of vital enzymes or rapid protein denaturation, which is immediately cytotoxic and leads to coagulative necrosis. ${ }^{7}$ At temperatures of around $40^{\circ} \mathrm{C}-45^{\circ} \mathrm{C}$, irreversible cell damage occurs only after prolonged exposure (from $30 \mathrm{~min}$ to $60 \mathrm{~min}$ ). At temperatures of above $60^{\circ} \mathrm{C}$, the time required to achieve irreversible damage decreases exponentially. Rising temperatures have been shown to change cell membrane fluidity and permeability, and this causes dysfunction of actin filaments and microtubules, leading to impairment of facilitated diffusion across the cell membrane. ${ }^{16}$ This process was first thought to be the main cause of hyperthermiainduced cell death. However, mitochondrial dysfunction has been shown to occur with heat-induced injury. ${ }^{17}$ Major sub-cellular structural changes can be seen minutes after heat injury; these include vesicularisation of the mitochondrial cristi, mitochondrial swellings and formation of dense bodies. ${ }^{18}$ DNA replication is also rapidly inhibited by hyperthermia. 


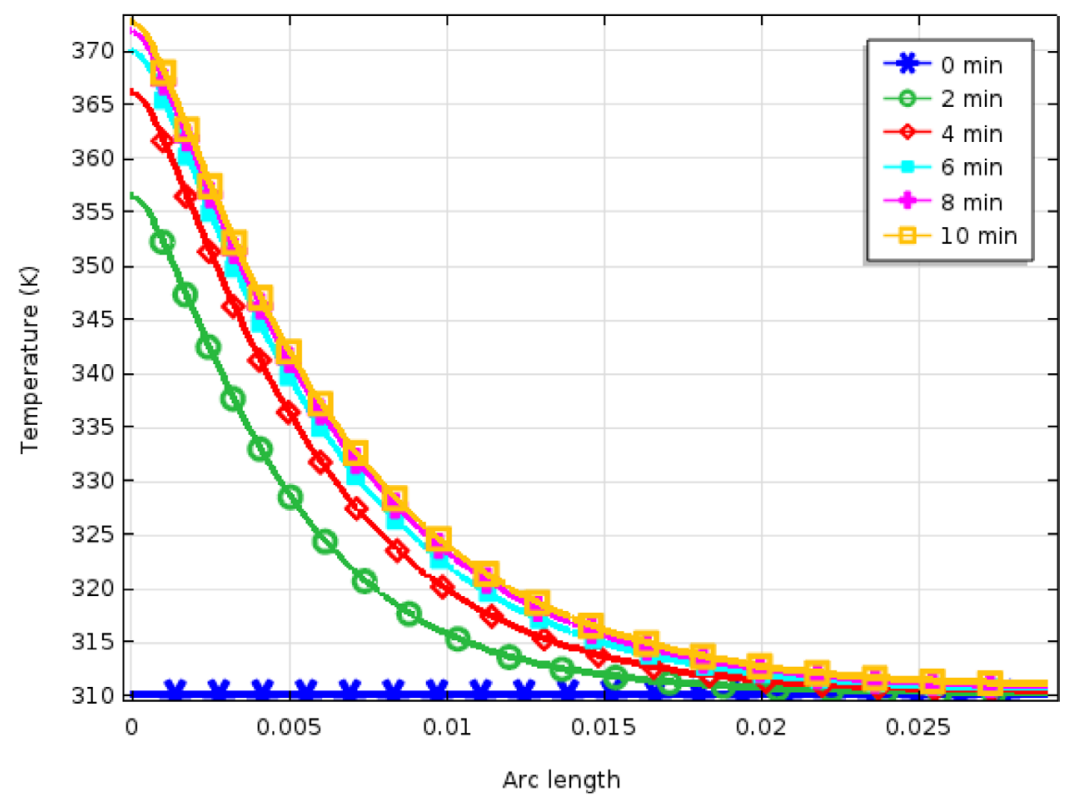

Figure 6: Effect of attenuation on tissue temperature.

The SAR evaluated at a distance of $2.5 \times 10^{-3} \mathrm{~m}$ parallel to the antenna axis is plotted in Figure 6. The SAR patterns of an interstitial antenna should be highly localised near the tip of the antenna which is clearly shown in Figure $7 .{ }^{8}$

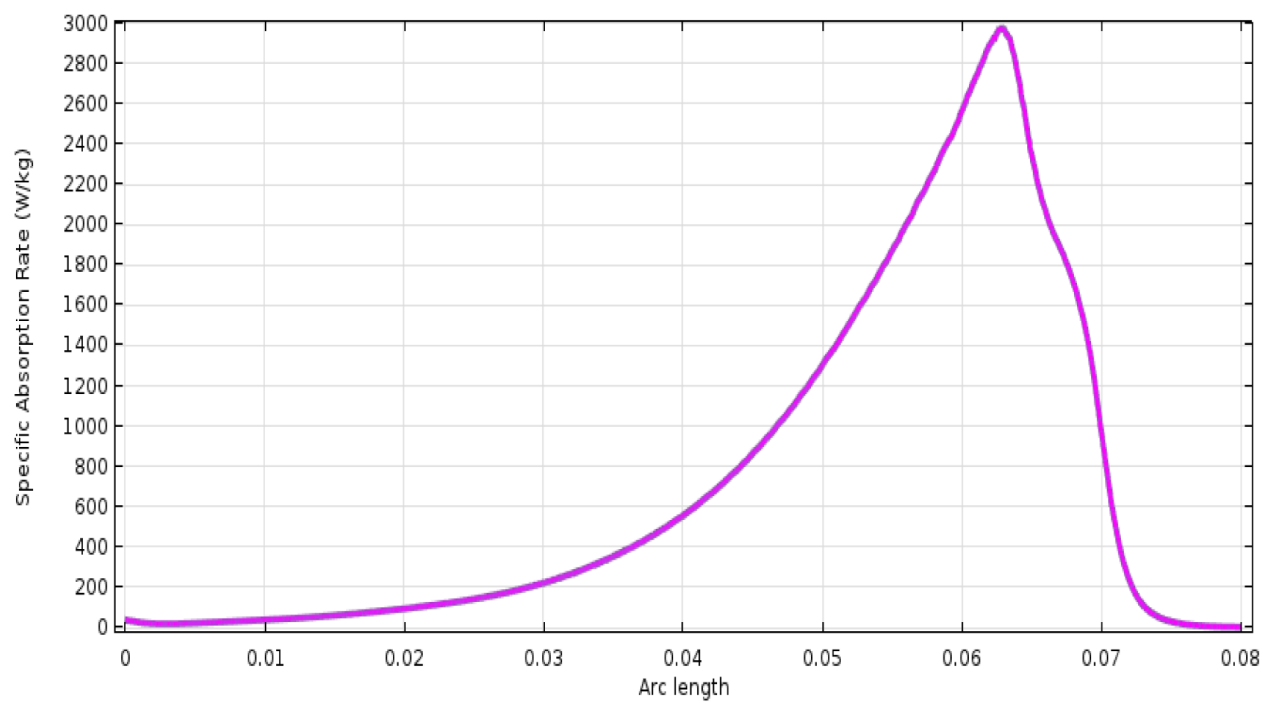

Figure 7: $\mathrm{SAR}$ in $\mathrm{W} / \mathrm{kg}$ along a line parallel to the antenna and at a distance $2.5 \mathrm{~mm}$ from the antenna axis. 
As seen in Figure 7, the SAR grows almost exponentially up till a point on the tissue corresponding to about $0.063 \mathrm{~m}$ of the arc described by the circular path of the emitted microwave energy. This exponential behaviour implies that the microwave power (energy over time) increases as it penetrates the tissue. This is because of the assumption that the tissue also contributes to the energy as the atoms along the path of the beam become thermally energised. This results in an increase in the overall energy supplied. The fall in the energy at about $0.063 \mathrm{~m}$ is as a result of the constant perfusion of the tissue by blood, thus reducing the energy of the microwave and attenuating this beam until it falls to its minimum value.

The fraction of necrotic tissue is visualised in the surface plot shown in Figure 8. The figures showcase that the fraction of necrosed tissue is higher at the antenna slot area. Comparing Figures 8(a) and (b), it can be seen that a larger fraction of the tissue near the slot is necrosed after 10 min compared with the amount of tissue necrosed in $5 \mathrm{~min}$. Thus, the amount necrosed is time dependent and the thickness of necrosed tissue increased with increasing time.
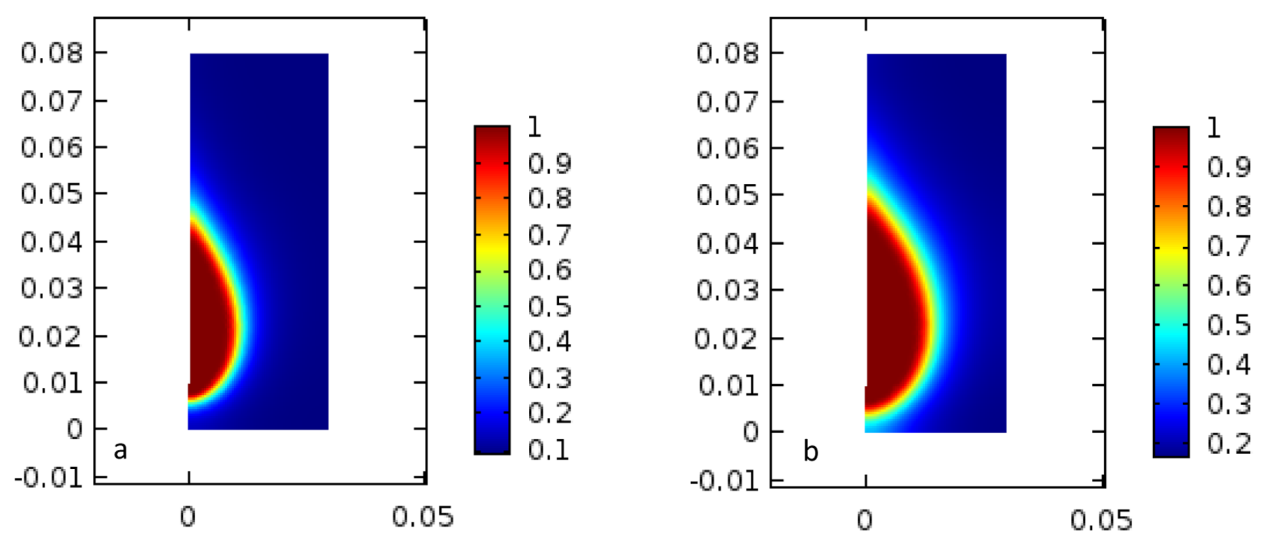

Figure 8: (a) Fraction of necrotic tissue after $5 \mathrm{~min}$ and (b) fraction of necrotic tissue after $10 \mathrm{~min}$.

The dark blue region of Figures 8 confirms that only about $10 \%$ of the tissue $3 \mathrm{~cm}$ away from the antenna is necrosed after $5 \mathrm{~min}$. Meanwhile, the figure shows that this doubles to $20 \%$ in $10 \mathrm{~min}$. This implies that the longer the time spent, the higher the necrosis. Further analysis on the dependence of fraction of necrosed tissue on attenuation and time was performed at four different points from the antenna slot. At a point $(0.02,0.005)$ from the microwave emitting slot of the antenna which falls in the region with $100 \%$ necrosis, the tissue is necrosed to about $100 \%$ in about $2 \mathrm{~min}$. Moving further to a point $(0.02,0.01)$ from the antenna, attenuation reduced the microwave energy supplied to the region and as a result the point is necrosed to about $80 \%$ after $5 \mathrm{~min}$ and to $100 \%$ in about $8 \mathrm{~min}$. The next point 
at $(0.02,0.015)$ corresponds to the domain which attains a maximum of a little over $60 \%$ necrosis in $10 \mathrm{~min}$. The fourth point at $(0.02,0.02)$ from the antenna slot shows that a little over $10 \%$ of the tissue is necrosed in 5 min whereas about $30 \%$ of the tissue is necrosed in $10 \mathrm{~min}$. Further analysis shows that rate of necrosis is not a linear relationship but has a logarithmic relationship with time. To reduce this effect, the heat is administered in fractions; shorter times spread over a period of days or weeks. In $5 \mathrm{~min}$, only about $8 \%$ of normal tissue is necrosed and about $16 \%$ of this is necrosed in $10 \mathrm{~min}$. Figure 9 show that tissue necrosis decayed exponentially as the heat passed through the tissue. The exponential decrease still results from field attenuation.

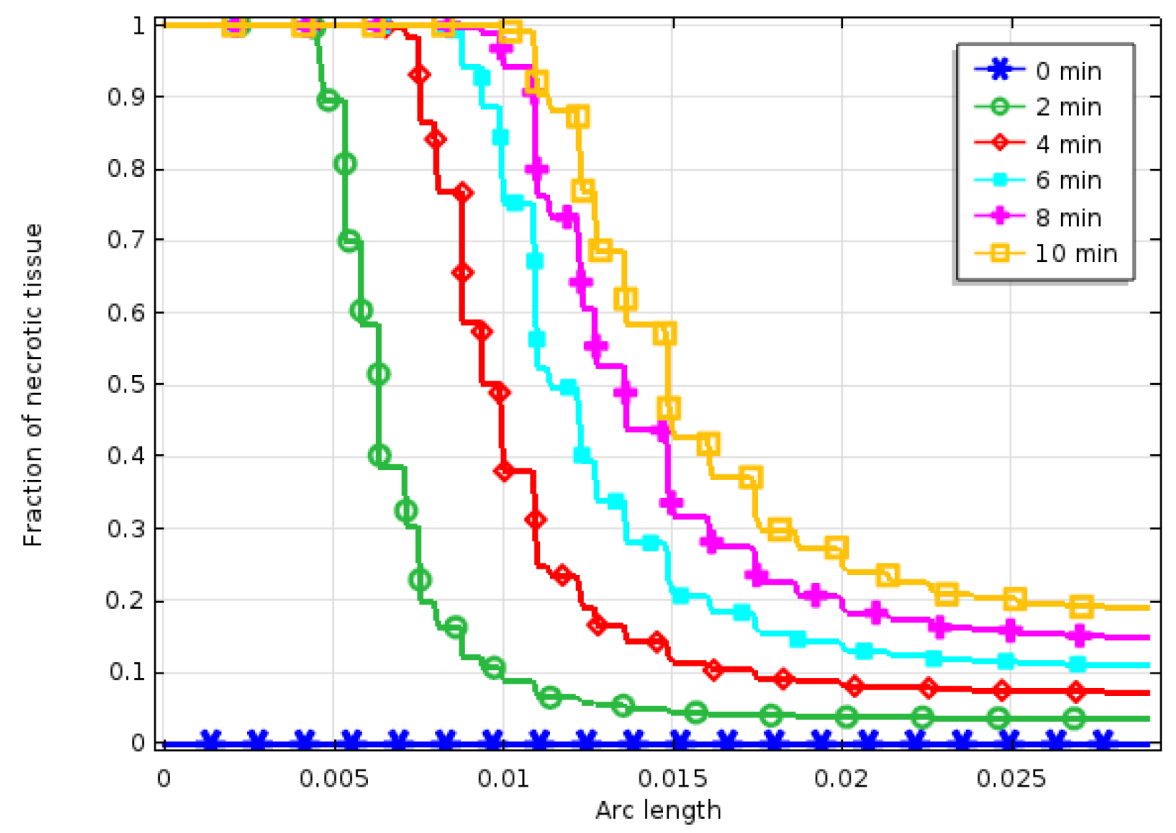

Figure 9: Fraction of necrotic tissue versus field penetration distance in meters.

In the transition zone, the region immediately surrounding the central area where coagulative necrosis takes place, studies have shown that the presence of inflammatory cells including neutrophils, macrophages, natural killer cells, dentritic cells as well as B and T cells specific to the ablated tissue. ${ }^{19-21}$

Mechanical cell damaged caused by hyperthermia causes the release of several intracellular substrates like Heat Shock Proteins (HSPs), DNA, RNA and High Mobility Group Protein B1 (HMGP1). ${ }^{22,23}$ All these generally lead to activation of humoral and cellular mediated antitumor immune response which is responsible for the indirect or delayed cellular damage. 
Indirect cellular damage, also referred to as delayed damage, occurs after thermal ablation by the release of pro-inflammatory cytokines such as interleukin-6 (IL-6), IL-8 and tumor necrosis factor- $\alpha$ (TNF- $\alpha)$. This has been shown in both preclinical and clinical studies and has several proposed mechanisms, including induction of apoptosis, vascular damage that causes ischaemia, ischaemia-reperfusion injury, lysosomal contents that are released during tumor necrosis or from invading granulocytes, cytokine release and further stimulation of an immune response. ${ }^{7,16}$

Input power has effect on some of critical parameters. As a result, input power has been varied to investigate its effect on the tissue necrosis. The plot of radial length of $100 \%$ necrotic tissue as a function of input power in Figure 10 shows a logarithmic relationship with a relation $d=0.054 \ln (P)-0.007$. where $d$ is thickness of $100 \%$ necrosed tissue and $P$ is the input power. This implies that the input power and time for the thermotherapy can be estimated if the volume density of the cancer tissue is known.

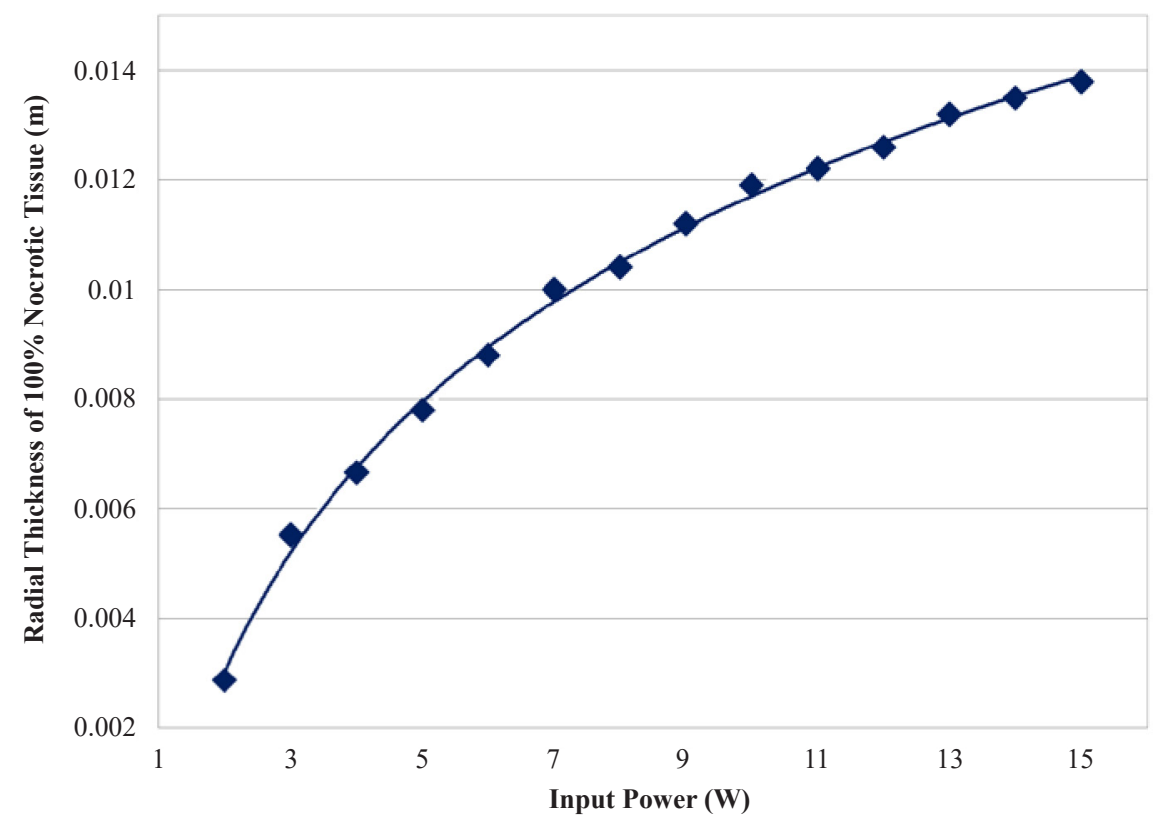

Figure 10: Thickness of necrosed tissue versus input power.

The total power dissipation is frequency dependent and as a result the tissue temperature and eventual tissue injury is frequency dependent. Analysis of the frequency dependence of necrotic layer will give the clinicians an idea of the operated frequency for optimum performance. The plot of radial length of $100 \%$ necrotic tissue as a function of frequency as shown in Figure 11 shows a fourth- 
degree polynomial relation. It appears that at frequency of $2.3 \mathrm{GHz}$, the level of increase in cell injury is not very significance. This suggest that $2.3 \mathrm{GHz}$ may be the appropriate operating frequency to perform a thermotherapy.

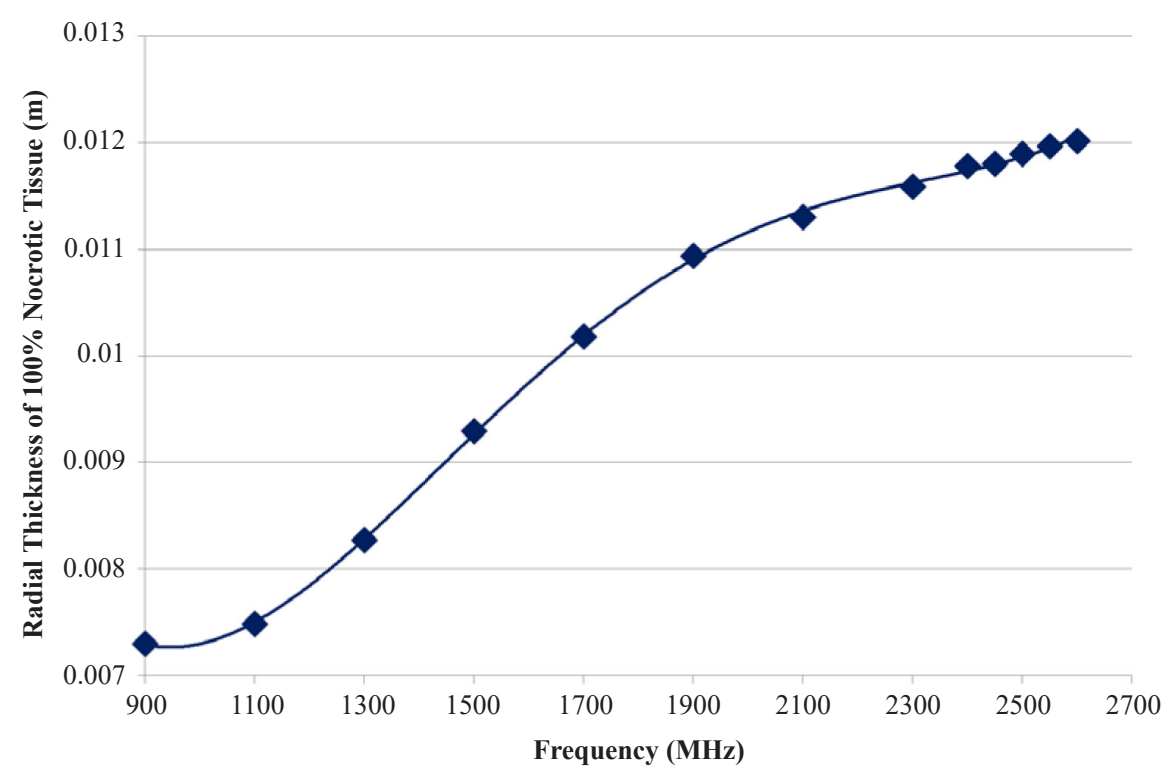

Figure 11: Thickness of necrosed tissue versus microwave frequency.

In terms of delayed or indirect mechanisms of tumour destruction, MWA is a weak stimulator of local inflammation, as well as innate and acquired antitumour immunity. The induction of pro-inflammatory cytokines, including IL1 and IL6 by MWA is minimal compared with that by the other ablative techniques, as is the expression of HSP70..$^{24,25}$ Even so, the extent of immune cell infiltrates in the ablated tissue is inversely correlated with clinical outcome - specifically, overall survival and risk of local recurrence. ${ }^{26}$

\section{CONCLUSION}

The observed higher temperature within the vicinity of the tissue around the slot results from the high electromagnetic field at the slot of the antenna. More power is dissipated at the region around the slot. The thermal energy decreases towards the domain of the tissue due to attenuation of the electromagnetic field as it travels through the tissue. The absorption of power by the tissue (SAR) is highly dependent on time and the input power of the antenna. The fraction of necrosis depends on input power, frequency, temperature of the liver and exposure time. Thus, input power, frequency and time area key factor in the hyperthermia. Higher power 
and/or longer times increase the injury caused by the antenna and can consequently damage healthy tissues leading to complications in normal tissues surrounding the liver. This suggests that care must be taken in the choice of microwave operating frequency and when varying the input power of the antenna.

\section{REFERENCES}

1. Chu, K. F. \& Dupuy, D. E. (2014). Thermal ablation of tumours: Biological mechanisms and advances in therapy. Nat. Rev., 14, 199-208, https://doi.org/ 10.1038/nrc3672.

2. Issadore, D. et al. (2009). Microwave dielectric heating of drops in microfluidic devices. Lab. Chip, 9, 1701-1706, https://doi.org/10.1039/ B822357B.

3. Bala, M. M. et al. (2013). Microwave coagulation for liver metastases (review). Cochr. Data. Syst. Rev., 10, https://doi.org/10.1002/14651858. CD010163.pub2.

4. Brace, C. L. (2009). Radiofrequency and microwave ablation of the liver, lung, kidney, and bone: What are the differences? Curr. Prob. Diag. Radiol., 38(3), 135-143, https://doi.org/10.1067/j.cpradiol.2007.10.001.

5. Stuerga, D. (2006). Microwave-material interactions and dielectric properties, key ingredients for mastery of chemical microwave processes. In Loupy, A. (Ed.). Microwave in organic synthesis, 2nd ed. Weinheim: WileyVCH Verlag, 1-61, https://doi.org/10.1002/9783527619559.ch1.

6. Haemmerich, D. et al. (2009). Electrical conductivity measurement of excised human metastatic liver tumours before and after thermal ablation. Phys. Meas., 30(5), 459-466, https://doi.org/10.1088/0967-3334/30/5/003.

7. Nikfarjam, M., Muralidharan, V. \& Christophi, C. (2005). Mechanisms of focal heat destruction of liver tumors. J. Surg. Res., 127, 208-223, https://doi.org/10.1016/j.jss.2005.02.009.

8. Berjano, E. J. (2006). Theoretical modeling for radiofrequency ablation: State-of-the-art and challenges for the future. Biomed. Eng., 5(24), 1-17. https://doi.org/10.1186/1475-925X-5-24

9. Bertram, J. M. et al. (2006) Antenna design for microwave hepatic ablation using an axisymmetric electromagnetic model. Biomed. Eng., 5(15), 1-9. https://doi.org/10.1186/1475-925X-5-15.

10. Buakaew, C. \& Tungjitkusolmun, S. (2008). Finite element analysis of microwave coagulation therapy of unwanted tissue for the treatment of snoring and obstructive sleep apnea. Paper presented at the 3rd International Symposium on Biomedical Engineering (ISBME), 447-450. 
11. Jiao, T. et al. (2012) A coaxial-slot antenna for invasive microwave hyperthermia therapy. J. Biomed. Sci. Eng., 5, 198-202, https://doi.org/ 10.4236/jbise.2012.54026.

12. Liu, A. J., Zhou, H. \& Kang, W. (2013). A numerical study on microwave coagulation therapy. Appl. Math. Sci., 7(104), 5151-5164, https://doi. org/10.12988/ams.2013.37392.

13. Pennes, H. H. (1948) Analysis of tissue and arterial blood temperatures in the resting human forearm. J. Appl. Physiol., 1, 93-122.

14. Laidler, K. J. (1984). The development of the Arrhenius Equation. J. Chem. Edu., 61(6), 494-498, https://doi.org/10.1021/ed061p494.

15. COMSOL. (n.d.). COMSOL Multiphysics, version 5.54.3, 2015. Retrieved from www.comsol.com on 15 January 2015.

16. Fajardo, L. F. et al. (1980). Effects of hyperthermia in a malignant tumor. Cancer, 45, 613-623, https://doi.org/10.1002/1097-0142(19800201)45:3\% 3C613::AID-CNCR2820450331\%3E3.0.CO;2-E.

17. Tiong, L. \& Maddern, G. J. (2011). Systematic review and meta-analysis of survival and disease recurrence after radiofrequency ablation for hepatocellular carcinoma. Br. J. Surg., 98, 1210-1224, https://doi.org/ 10.1002/bjs.7669.

18. Wheatley, D. N., Kerr, C. \& Gregory, D. W. (1989). Heat-induced damage to HeLaS3 cells: Correlation of viability, permeability, osmosensitivity, phase-contrast light-, scanning electron- and transmission electronmicroscopical findings. Int. J. Hyperth., 5, 145-162, https://doi.org/ $10.3109 / 02656738909140444$.

19. Dromi, S. A. et al. (2009). Radiofrequency ablation induces antigenpresenting cell infiltration and amplification of weak tumor-induced immunity. Radiol., 251, 58-66, https://doi.org/10.1148/radiol.2511072175.

20. Wisniowski, T. T. et al. (2003). Activation of tumor-specific T lymphocytes by radio-frequency ablation of the VX2 hepatoma in rabbits. Cancer Res., 63, 6496-6500.

21. Zerbini, A. et al. (2010). Radiofrequency thermal ablation for hepatocellular carcinoma stimulates autologous NKcell response. Gastroent., 138, 19311942, https://doi.org/10.1053/j.gastro.2009.12.051.

22. den Brok, M. H. M. G. M. et al. (2004). In situ tumor ablation creates an antigen source for the generation of antitumor immunity. Cancer Res., 64, 4024-4029, https://doi.org/10.1158/0008-5472.CAN-03-3949.

23. Sabel, M. S. (2009). Cryo-immunology: A review of the literature and proposed mechanisms for stimulatory versus suppressive immune responses. Cryobiol., 58, 1-11, https://doi.org/10.1016/j.cryobiol.2008.10.126. 
24. Ahmad, F. et al. (2010). Changes in interleukin $1 \beta$ and 6 after hepatic microwave tissue ablation compared with radiofrequency, cryotherapy and surgical resections. Am. J. Surg., 200, 500-506, https://doi.org/10.1016/j. amjsurg.2009.12.025.

25. Ahmad, F. et al. (2010). Renal effects of microwave ablation compared with radiofrequency, cryotherapy and surgical resection at different volumes of the liver treated. Liver Int., 30, 1305-1314, https://doi.org/10.1111/j.14783231.2010.02290.x.

26. Dong, B. W. et al. (2003). Sequential pathological and immunologic analysis of percutaneous microwave coagulation therapy of hepatocellular carcinoma. Int. J. Hyperth., 19, 119-133, https://doi.org/10.1080/0265673 021000017154 . 